\title{
Ice Accretion Effects on Helicopter Rotor Performance, via Multi-body and CFD Approaches
}

\author{
Daniel Kelly ${ }^{1}$, Wagdi G. Habashi ${ }^{2}$ \\ CFD Laboratory McGill University, Montreal, Quebec, H3A 2S6, Canada \\ Giuseppe Quaranta ${ }^{3}$, Pierangelo Masarati ${ }^{4}$ \\ Politecnico di Milano, Milano, 20135, Italy \\ Marco Fossati ${ }^{5}$ \\ Aerospace Centre of Excellence, University of Strathclyde, Glasgow, G1 1XJ, United Kingdom
}

A numerical approach for assessing the degraded aerodynamics and flight characteristics of icecontaminated helicopter rotors is proposed. A hybrid two- and three-dimensional loose coupling strategy between Multibody Dynamics modeling and Computational Fluid Dynamics icing is formulated that attempts to balance computational resources, model complexity and accuracy for use during the early-design phases. A quasi-3D formulation that considers the heat transfer and the motion of the water film due to centrifugal effects is introduced. The method is suited for the analysis of rime, glaze and/or mixed ice conditions. Degraded aerodynamic and dynamic characteristics of the iced rotor and the changes in flight performance are assessed. The technique has been applied to the scenario of isolated helicopter rotors in hover and in forward flight. Deterioration of the figure of merit is also presented.

1 Graduate student, Dept. of Mechanical Engineering, 688 Sherbrooke St. W., AIAA Member.

2 Professor, Director, Dept. of Mechanical Engineering, 688 Sherbrooke Street West, AIAA Fellow.

3 Associate Professor, Dept. of Aerospace Science and Technology, via La Masa 34, AIAA Member.

4 Associate Professor, Dept. of Aerospace Science and Technology, via La Masa 34, AIAA Member.

5 Lecturer and Chancellor's Fellow, Dept. of Mechanical and Aerospace Engineering, 75 Montrose street, AIAA Member.

Peer-reviewed accepted author manuscript of the following article: Kelly, D., Habashi, W. G., Quaranta, G., Masarati, P., \& Fossati, M. (2018). Ice accretion effects on helicopter rotor performance, via multibody and CFD approaches. Journal of Aircraft, 55(3), 1165-1176. DOI: 10.2514/1.C033962 


\section{Nomenclature}

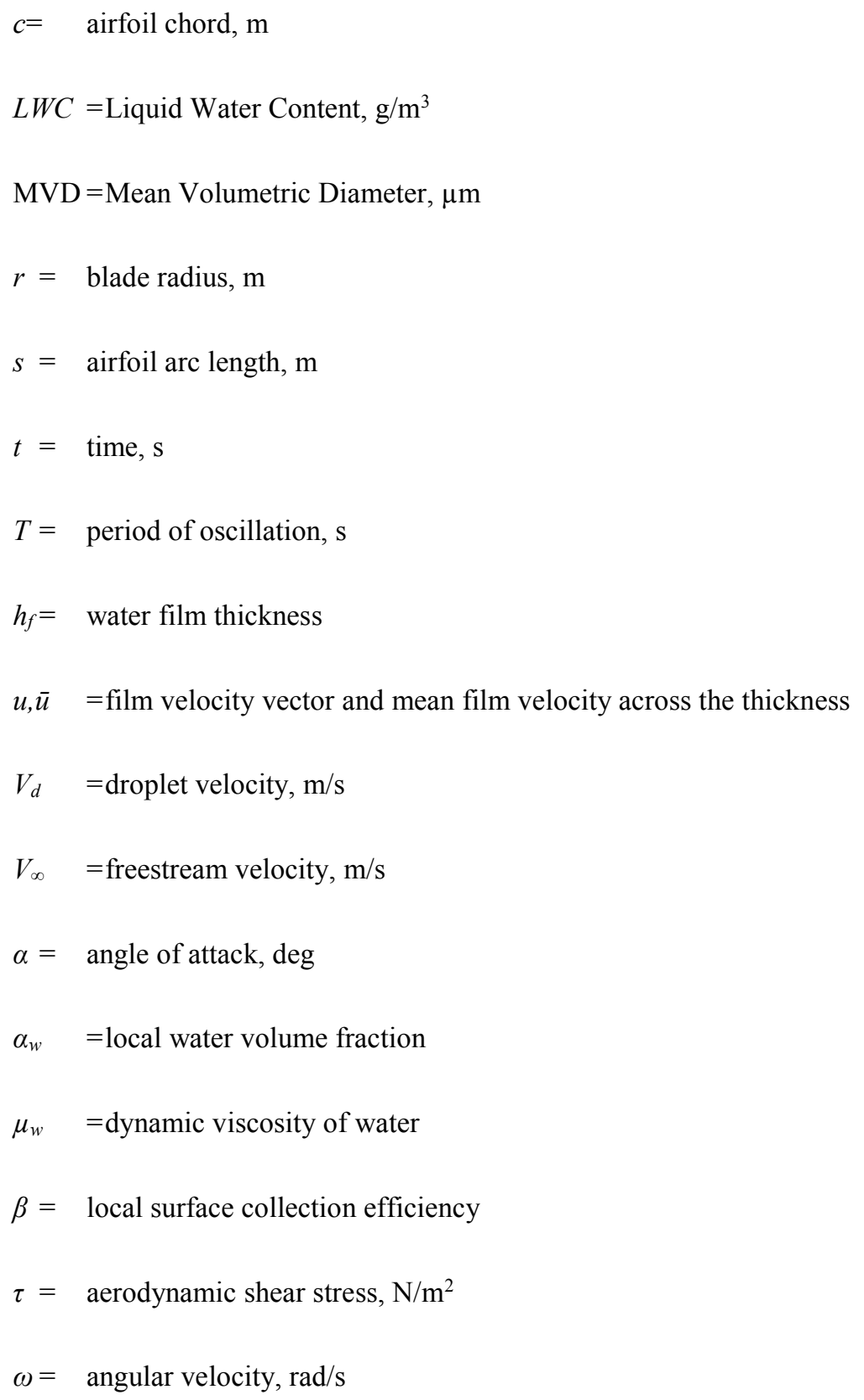

\section{Introduction}

Ice accretion represents a problematic scenario for helicopters. Ice deposited on rotor blades alters the airfoil geometry and increases surface roughness. Drag is increased, while transient and/or local airfoil stall may occur. The presence of ice may pose additional mechanical concerns due to the increased mass and altered aerodynamic loading. Imbalances in the rotor may result in changes to the critical vibration characteristics and/or mechanical loading of 
rotor components. This can play a major role in safety and/or mission success, and thus it is important to take the development of an ice protection system (IPS) into consideration from the very early design phases. Due to the unique aerodynamics, dynamics and structure of helicopter rotors, the development of IPS for rotorcraft cannot simply adapt the existing IPS designs developed for fixed-wing aircraft [1]. Methodologies and approaches targeted with a specific application towards helicopters must to be considered.

Aiming for all-weather, fully-capable IFR operations, a reliable assessment of the impact of ice accretion on helicopter aerodynamics, dynamics and performance from the early design phases is a cost-effective way to address the problem in a timely manner. This will allow to: a) determine the requirements of the system, in terms of protection limits and power required, and b) determine the severity of icing on the helicopter should the protection system fail.

Traditional means of evaluating the impact of ice on helicopters involve experimental testing in natural, tanker or wind tunnel-based settings [2]. Changes in flight characteristics and performance degradation for model and full-size rotors in hover and forward-flight configurations have been reported in recent literature [3-6]. All these methods, however, are best suited for the later design phases that come after many crucial design decisions have been made, the change of which may have a huge impact on the cost and time to market.

Recent advances in numerical approaches have made it possible to adopt computational tools that complement and assist experimentation [7]. A requirement of modern aeroicing tools for rotorcraft includes the ability to predict not only the ice geometry, but also performance metrics and adjustments in helicopter rotor dynamics resulting from an icing encounter. Approaches exist in the literature that address icing on helicopter rotors via coupled interactions between a CFD ice accretion code (CFDI) and a multibody-dynamics model (MBD), like that proposed by Rajmohan, and collaborators [8]. Their method provided fair matching of torque rise in icing for an experimental UH-60A helicopter rotor in forward flight through the combined use of DYMORE, GT-Hybrid and LEWICE. A different loosely-coupled approach, proposed by Narducci, et al demonstrated good agreement for hover and forward flight test cases by adopting a combination of OVERFLOW, RCAS and LEWICE [9, 10]. While both approaches provide good agreement for ice geometry and rotor performance degradation, they come with the high computational expense of 3D airflow and droplet solutions coupled to structural model dynamics.

The complexity of these existing approaches, in terms of model setup and computational cost, make them unsuitable for the early design phases. During the early design phases of a helicopter, diverse design solutions may be 
evaluated and the rotor's design may undergo frequent adjustments. Rapid design iterations and feedback from modeling techniques are required. IPS and/or icing certification may form an integral part of the design of the rotor, requiring an evaluation of the rotor's performance in icing conditions, but detailed geometric information about the rotor or blades may be limited. For these reasons, a lower-cost model may be more suitable.

Moreover, while the existing methodologies adopt 3D rotor aerodynamic solutions, ice accretion is only performed over 2D sections via a strip-theory approach. This may limit the capability of these models to evaluate glaze ice conditions, where droplets impinging on the surface may not freeze instantly but coalesce to form a film of liquid water. This water film may migrate along the surface before freezing, eventually producing non-aerodynamic shapes characterized by horns of ice, and/or freezing over unprotected sections of the rotorcraft. Significant motion of the water film can occur due to aerodynamic forces and, especially in the case of helicopter rotors and propeller blades, as a consequence of centrifugal effects. By using $2 \mathrm{D}$ icing approaches, the motion of water along the blade and the radial heat exchange cannot be addressed properly, with consequent effects on the thermodynamics of ice accretion in the case of glaze icing and/or in the case of Supercooled Large Droplets (SLD) icing environments. Such scenarios demand increased simulation fidelity that can be obtained via 3D icing approaches.

The proposed methodology aims to accelerate the development of helicopter IPS by improving the capability of assessing 3D icing on rotors from the early design phases. The evolution of a recently proposed loosely-coupled MBDCFDI approach [11] is presented that aims at addressing the problem of 3D icing and its consequences on rotor dynamics. The methodology attains a mid-point in terms of cost and accuracy between entirely $2 \mathrm{D}$ and 3D computations. The approach adopts a combination of 2D and 3D methodologies that reverses the commonly used aeroicing approach for rotor blades: a 2D approach is used for the aerodynamic and droplet impingement solutions, while a 3D approach is used for icing.

The rationale is that the actual motion of the water along the blade span and the heat transfer between adjacent blade sections is numerically accounted for during the icing phase. Observations from experimental campaigns conducted by Tanner and Yaggy, [17], and by McCroskey [18], for hover and forward flight, support this approach by demonstrating airflow patterns on the surface of the rotor blades that were mainly $2 \mathrm{D}$. In airflow regions away from the blade tip, boundary layer growth was observed to be almost exclusively $2 \mathrm{D}$ in both rotating and stationary frames, with rotational effects having only a minor influence on cross-flow. Centrifugal effects had, however, a 
noteworthy influence on the motion of an impinged liquid film along the blade, demonstrated by oil film flow at directions up to $90^{\circ}$ from the chord line [18].

Another crucial aspect that needs to be taken into consideration while considering IPS design for helicopters is the difference between icing in hover or forward flight conditions. The very different aerodynamic environment that characterizes these two operating regimes result in different ice shapes and consequently different dynamic responses of the iced rotor. In order represent accurately the time-varying aerodynamics of the forward flight condition, it is important to account for the cyclic pitch motion of the blades and represent accurately the combination of the blade's motion and advancement velocity of the rotorcraft. In a $2 \mathrm{D}$ framework, this situation is typically accounted for by adopting a pitching motion for the blade's section according to the rotor's angular velocity and the section's radial location. Nevertheless, by accounting only for the change in the pitch over the azimuthal position, nothing is done to address the difference between the velocity of the air encountering a section of the advancing blade and that encountering a section on the retreating blade. This circumstance has an impact also on the droplet dynamics and consequently on water deposition over the blade surface. In the proposed approach, the standard 2D pitching airfoil paradigm is extended to properly account for these differences on the flow velocity during forward flight conditions.

First, the methodology is introduced as a general framework, along with a discussion of the solvers presently implemented. A quasi-3D technique for the calculation of water film motion and ice growth on the blade surface is then proposed, as well as an unsteady aerodynamic evaluation technique for forward flight. Next, evaluation of the numerical model is done by comparison to two icing tunnel test cases; one for a rotor in hover flight, and another for a rotor in forward flight at a moderate advance ratio. A discussion compares the results obtained from the simulation against available experimental data.

\section{Methodology}

The loose-coupled approach is outlined in Fig. 1. A trimmed flight condition is first established through the use of a multi-body approach for rotor dynamics (MBDyn is used here [12]). The blade aerodynamics, in terms of angle of attack (AoA) and incident velocity, is passed to an aeroicing module (FENSAP-ICE [15]), which simulates the accretion of ice and assesses aerodynamic degradation. The new trim condition for the ice-contaminated rotorcraft is eventually assessed via the multi-body module. The degraded performance characteristics of the rotor, in terms of thrust loss and torque rise, are determined via a comparison of clean and iced rotor dynamic models. A quasi-steady 
multishot approach is adopted to advance the simulation in small time intervals, as selected by the user, such that the unsteady growth of the ice geometry and its impact on aerodynamic calculations can be reproduced in a realistic manner.

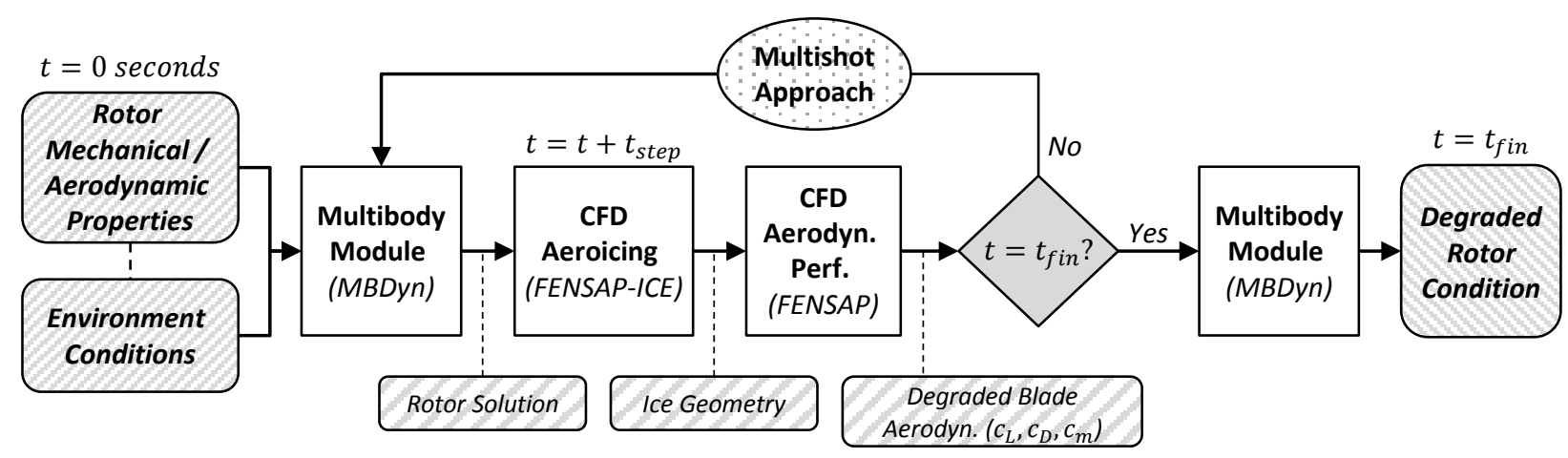

Fig. 1 Loose-Coupling Approach for Aeroicing on Isolated Rotors

\section{Structural Dynamics and Performance Modeling}

Rotorcraft dynamics and performance are evaluated through the use of a comprehensive multibody analysis.

The present work makes use of the general-purpose multi-body solver MBDyn [12]. Initially, an isolated model of the helicopter rotor is assembled within the multibody context, formulated as nodes and elements in a nonlinear Finite Element fashion. A hub, incorporating pitch links, swashplates, flap, pitch and lead-lag hinges, joins each blade. The blade's structural properties are modeled through the use of rigid bodies. Blade airfoil geometry is specified at radial sections representing the geometric variations along the span.

Aerodynamic data are made available to the MBD module via lookup tables of aerodynamic coefficients obtained through 2D CFD solutions over different blade sections. A blade-element momentum theory (BEMT) model is used to determine the distribution of the inflow along the blade span, providing as output key variables including AoA and incident velocity along the blade span. When considering a helicopter in forward flight, a linear inflow model as proposed by Drees is employed [13], with corrections for unsteady blade flapping aerodynamics applied according to Theodorsen's theory [13]. A trimmed condition is reached through an iterative approach, adjusting collective and cyclic inputs to realize balanced forces on the rotor disc in the desired flight condition. Rotor kinematics and blade motion are then recorded for one blade rotation, specifically describing flap motion, pitch and induced velocity at the selected radial sections. 
After each shot of the icing process, the aerodynamic lookup table is updated with new values for the coefficients of the ice-contaminated rotor. An additional degradation factor, surface roughness, is applied through a model that approximates ice growth as hemispherical beads [14]. The inertia attributed to the growth of ice on the blade is considered by including several point masses along the blade at the appropriate chord-wise locations.

\section{Aerodynamics / Aeroicing Module}

The module adopted for flow, droplet impingement and icing is FENSAP-ICE [15]. This module follows a threestep procedure, successively calculating airflow, droplet impingement and ice accretion. An Eulerian approach is used to compute the dynamics of water droplets suspended within the airflow. Water deposition is quantified in terms of the so-called collection efficiency, $\beta$,

$$
\beta=\frac{-\alpha \overrightarrow{V_{d}} \cdot \vec{n}}{L W C * V_{\infty}}
$$

The ice accretion module performs a thermal analysis of the multiphase air-water-ice scenario on the blade surface. The velocity and path of the water film is determined as a result of the aerodynamic shear stress and centrifugal effects. Here, a thin water film is assumed (on the order of microns) and an average water film velocity is obtained.

$$
\begin{gathered}
u(x, y) d y=\frac{h_{f}}{2 \mu_{w}} \tau_{w}(x) \\
u(x, y)=\frac{1}{h_{f}} \int_{0}^{h_{f}}
\end{gathered}
$$

The quantity and rate of freezing, evaporation and/or sublimation of the water film are applied through conservation laws. If freezing is predicted, an incremental growth in the geometry occurs $[15,16]$.

\section{Quasi-3D Approach}

The quasi-3D approach, referred to as Hybrid 2D/3D, is outlined in Fig. 2. The blade is initially discretized into 2D sections along the blade span. The local airfoil geometry is extracted at each section, and a 2D mesh is generated, with adjacent sections stitched to create a 3D mesh for the helicopter blade surface. A multiphase airflow and droplet impingement simulation is conducted in $2 \mathrm{D}$ at each selected blade section. Boundary conditions are applied to represent the local AoA and incident velocity, as obtained from the dynamic rotor solution. 3D fields of aerodynamic quantities and droplet dynamics are generated from the series of 2D airflow and droplet impingement simulations by interpolation over the 3D blade surface. Values for shear stress and heat transfer coefficient at the airfoil surface are 
extracted from the airflow simulation, while surface collection efficiency and droplet velocity are obtained from the droplet simulations.

The 3D blade surface mesh and fields for airflow and droplet impingement enable the calculation of ice accretion in 3D. The mesh is rotated during the icing process, allowing for spanwise motion of the impinged liquid film as driven by centrifugal forces. Aerodynamic shear stress drives the liquid film exclusively in the chordwise direction due to the $2 \mathrm{D}$ airflow calculations. The $3 \mathrm{D}$ mesh connectivity additionally permits heat transfer in the spanwise direction through conduction.

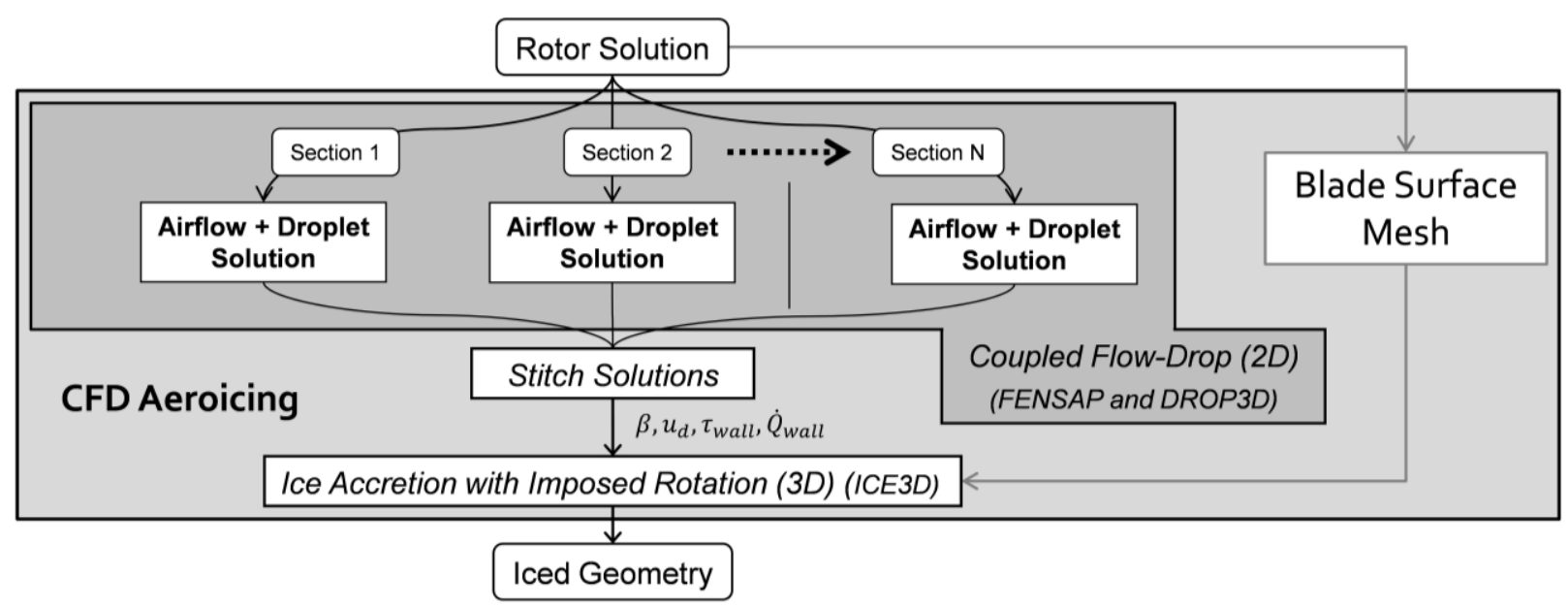

Fig. 2 Hybrid 2D/3D process for ice accretion

After ice is obtained in a 3D fashion, the ice shape at the selected 2D sections is obtained by performing interpolation among the two nodes of the 3D mesh closest to the plane at the specified radial distance. Once the 2D ice shapes are reconstructed, mesh deformation is performed. In case of excessively irregular ice shapes (big horns or feathers, or change in the geometric topology), the mesh is regenerated entirely to ensure the grid density at the solid wall is equal to the original airfoil mesh and a high level of resolution is retained. The aerodynamic coefficients representative of the iced blade section are obtained through a variable sweep of angle of attack and velocity.

\section{Unsteady Aerodynamics in Forward Flight}

In forward flight, a periodically-varying aerodynamic environment is generated due to the advancing velocity of the helicopter. An advancing and retreating blade side is identified on the rotor, where the local incident velocities of the air are higher and lower than the rotational velocity, respectively. To maintain a balanced load on the rotor disc, 
the pilot applies cyclic inputs to impose pitch changes around the azimuthal angle. These variations may have a profound impact on the aerodynamic condition of the blades, affecting shear stress values and droplet impingement locations.

In order to account for these effects in the numerical simulation, an unsteady approach has been selected. The inflow values for the selected 2D sections are initially obtained via the multibody approach. The resulting blade dynamics are then modeled on 2D blade sections as geometric pitching / flapping, and velocity changes according to the forward velocity of the helicopter. 2D airfoil motion is expressed in terms of time-varying functions for angle of attack and incident velocity. A sinusoidal model closely approximates the oscillation in these variables:

$$
\begin{aligned}
& \alpha_{2 D}=\alpha_{a v g}+\alpha_{u s} \sin \left(\frac{2 \pi}{T} t\right) \\
& V_{2 D}=\omega r+V_{\infty} \sin \left(\frac{-2 \pi}{T} t\right)
\end{aligned}
$$

An unsteady multiphase airflow / droplet impingement simulation is conducted through a dual time-stepping approach, marching in time through a second-order Gear scheme. Oscillation of the mesh is achieved through an ALE formulation. The true frequency of the blade rotation is maintained for the airflow and droplet impingement calculations. Several oscillation periods are computed until a periodic solution is reached [19].

A number of solution snapshots are extracted at discrete, evenly spaced intervals along one period of oscillation for the converged solution. At each snapshot ice accretion is computed for a small time step, usually in the order of 0.001 seconds, thus emulating the dynamic blade motion / aerodynamic effects. The ice accretion process is repeated through several oscillation periods until the desired ice accretion time is reached. Figure 3 summarizes the discretized ice accretion steps for one oscillation period, extracted from the unsteady aerodynamic solutions. 


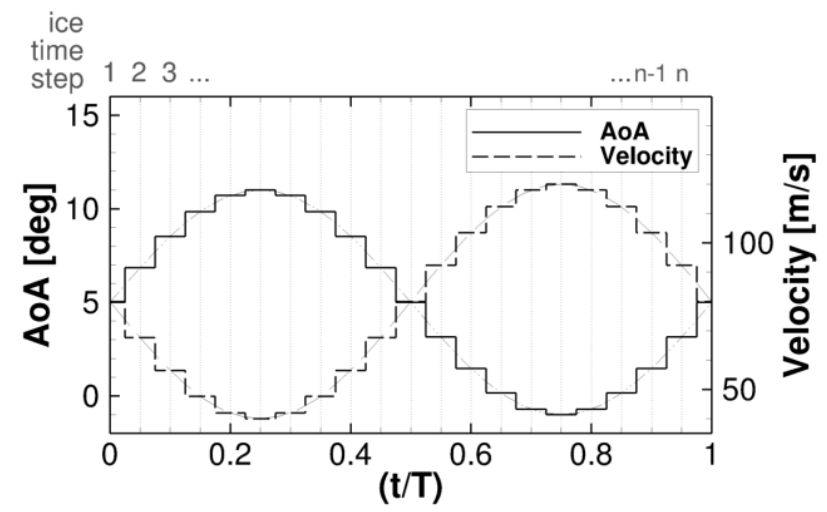

Fig. 3 One rotation period, oscillations in angle of attack (upper) and velocity (lower)

The unsteady aerodynamic approach has been merged with the hybrid 2D/3D approach to perform quasi-3D ice accretion calculations in forward flight. A 3D surface mesh is generated from 2D blade sections, and 3D airflow / droplet impingement solution files are generated for each snapshot from the 2D solutions at selected blade sections. Ice accretion is simulated at discrete time steps until the icing time period is obtained. Figure 4 demonstrates the hybrid $2 \mathrm{D} / 3 \mathrm{D}$ process as applied to unsteady forward flight.

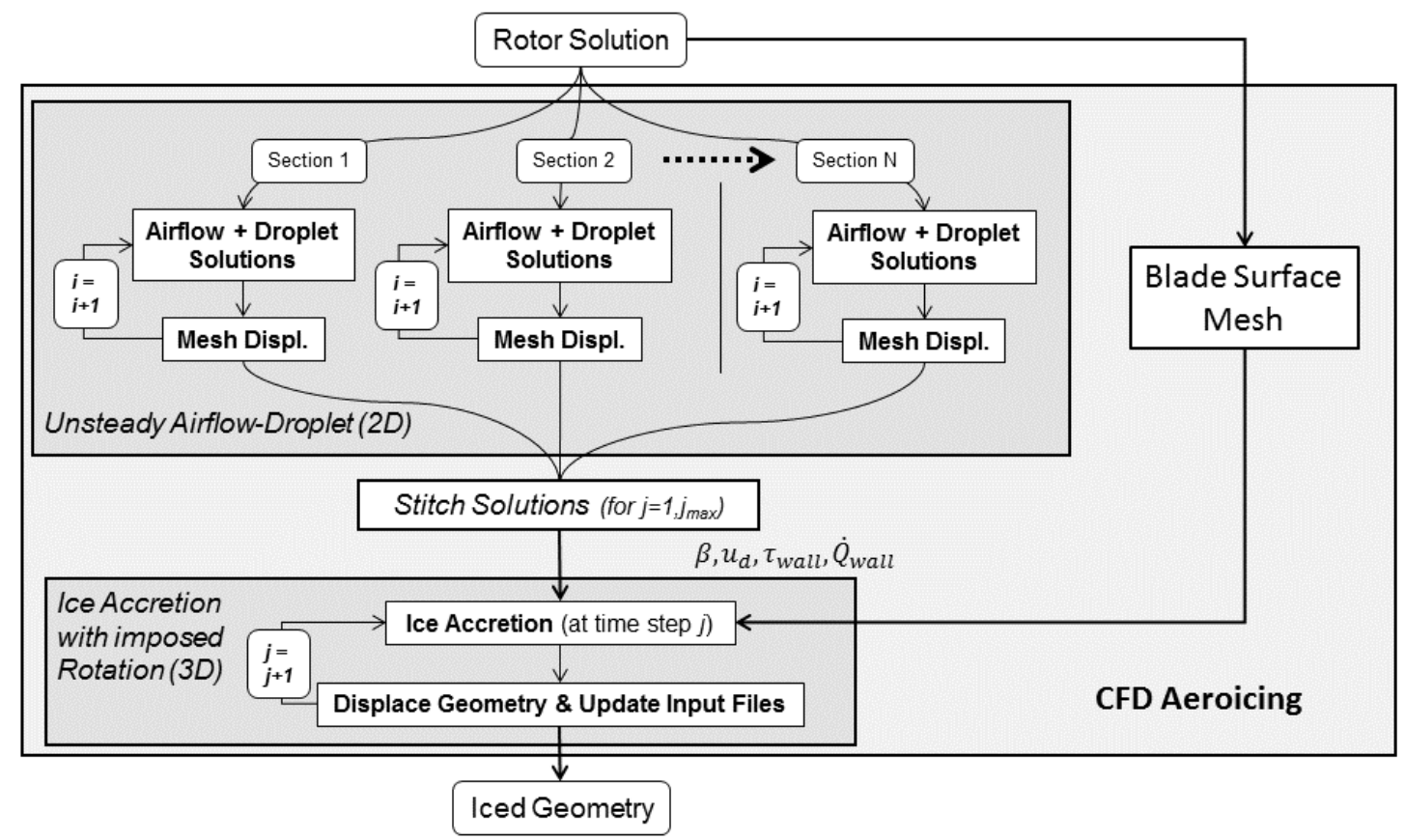

Fig. 4 Sequence diagram for the Hybrid 2D/3D process in forward flight 


\section{Hover Flight Case}

The first test case considers a rotor in hover conditions. The Adverse Environment Rotor Test Stand (AERTS) program was selected as a source of reference data $[3,20]$. This series of tests evaluated icing effects on an instrumented, model-sized rotor inserted in a refrigerated test chamber. The two-bladed rotor features rectangular blades, a diameter of $2.36 \mathrm{~m}$, a NACA0015 airfoil profile, a chord of $0.172 \mathrm{~m}$ and 2.6 degrees of linear twist. Measured parameters include ice geometry at several blade sections, and a rise in torque. The rotational speed of 600 RPM used in these experiments corresponds to a tip velocity of $74 \mathrm{~m} / \mathrm{s}$. This velocity is slower than the tip velocity on full-size helicopters, and as a result the aerodynamic and centrifugal forces encountered will be lower than for real-world operations. However, this test case is presented as a means of demonstrating the performance of the proposed approach in hover flight.

Liquid water droplets are introduced into the chamber via ceiling-mounted nozzles. Due to unequal mixing within the test chamber, a non-constant LWC distribution has been observed, with reduced LWC values at the blade root sections. MVD is reported to be monodispersed, with the atomizing nozzles calibrated to $\pm 2 \mu \mathrm{m}$ [3]. Run 44 provided mixed icing conditions for evaluation, with the blade tip encountering glaze icing conditions due to aero-thermal heating. No shedding has been noted in this run. Environmental conditions are reported in Table 1:

Table 1 Experimental Conditions for AERTS Run 44

\begin{tabular}{lcc}
\hline Parameter & Value & Units \\
\hline Tip Speed & 74.14 & $\mathrm{~m} / \mathrm{s}$ \\
Rotor Speed & 600 & $\mathrm{RPM}$ \\
Collective & 2.5 & $\mathrm{deg}$ \\
Static Temp. & -10.1 & ${ }^{\circ} \mathrm{C}$ \\
LWC (@ Tip) & 1.3 & $\mathrm{~g} / \mathrm{m}^{3}$ \\
MVD & 15 (Monodisp.) & $\mu \mathrm{m}$ \\
Icing Time & 180 & $\mathrm{sec}$ \\
\hline
\end{tabular}

The blade has been discretized into 24 sections, representing a variation in incident velocity of less than $2 \mathrm{~m} / \mathrm{s}$ between adjacent blade sections. 2D meshes are generated at each blade section, and a fully-articulated rotor is modeled in MBDyn. Due to the short blade span, blades are approximated as rigid-body elements. An aerodynamic database has been created for the NACA0015 profile through steady 2D calculations at angles of attack from $-6^{\circ}$ to $+14^{\circ}$. The dynamic model has been evaluated at a range of rotor speeds to generate a performance map of the rotor. 
Model performance, in terms of rotor torque, is shown to match experimental data in the literature, illustrated in Fig. 5. Blade collective pitch was held constant at 2.5 degrees throughout the encounter.

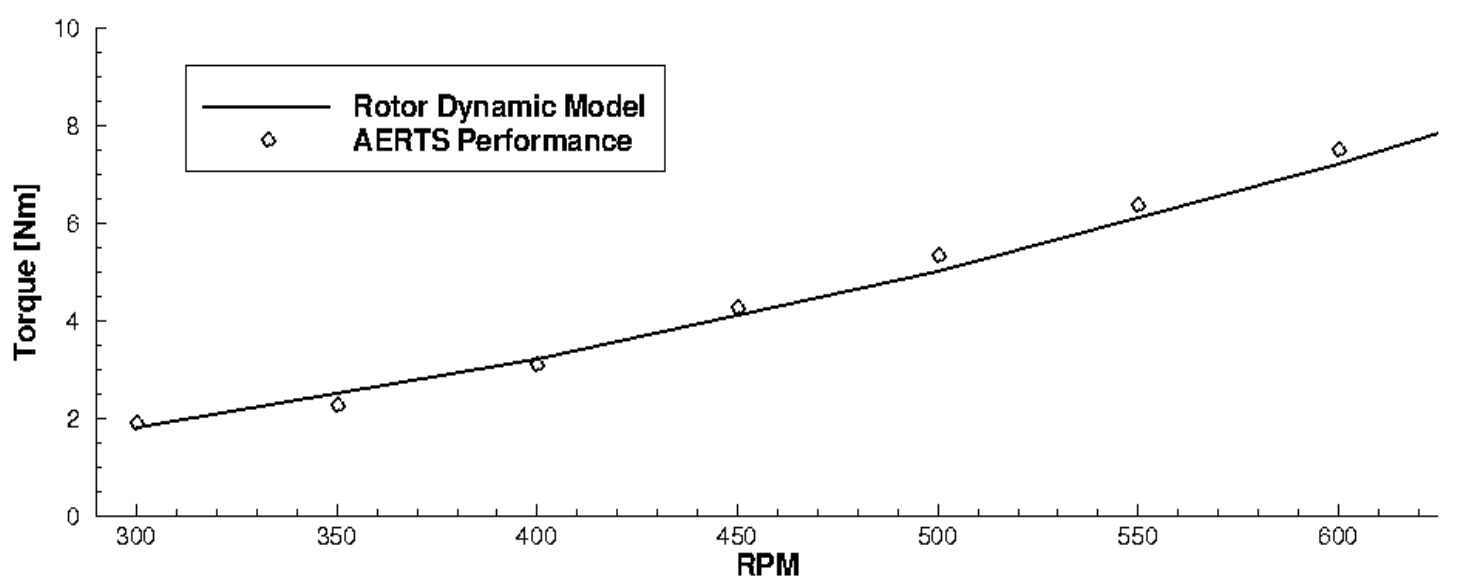

Fig. 5 Performance of the rotor dynamic model evaluated with experimental data for the AERTS rotor

Inflow characteristics, defining the angle of attack and the incident velocity distribution along the blade span, he been extracted from the dynamic model at rotor / hub conditions representing experimental run 44. Hybrid 2D meshes of 64,000 elements were generated for each section. These meshes contain 35 structured layers, and a maximum surface $\mathrm{y}^{+}$of 1 observed in the first structured layer. Figure 6 illustrates a 2D mesh of the clean NACA0015 as used in the ice accretion process.
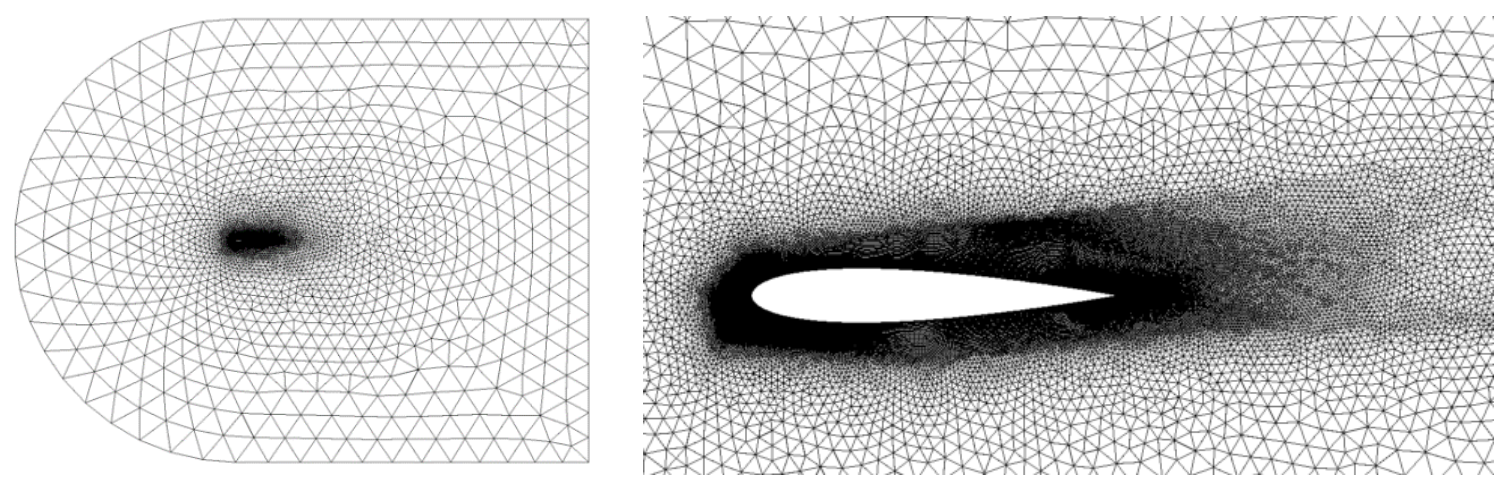

Fig. 6 Illustrations of the 2D hybrid NACA0015 mesh [21]

\section{Water Film and Aeroicing Results}

Ice geometries have been computed using two approaches: a) ice accretion on isolated 2D sections, and b) ice accretion through the hybrid 2D/3D approach. Both approaches are assessed using a 6-shot approach, with re-meshing between shots. 
Chordwise and spanwise components of water film velocity are provided as computed using the Hybrid 2D/3D approach for the AERTS test case in Fig. 7. CFDI calculations show an increase in local velocity along the blade span promotes a coalescence of water droplets into a film layer outboard of $0.9 \mathrm{~m}(76 \%)$ span; instantaneous freezing of water droplets is calculated near the blade root. Chordwise water film velocity is noted to reach a maximum value at the airfoil upper and/or lower surface; a minimum chordwise velocity value is obtained near the stagnation point. Conversely, the spanwise component of water film velocity is shown to reach a maximum value near the stagnation point. The maximum spanwise water film velocity is found to be $30 \%$ of the maximum chordwise film velocity. The quasi-3D results predict a slight pooling of water film thickness at the blade tip, while a reduction in water film thickness is noted at inboard sections.

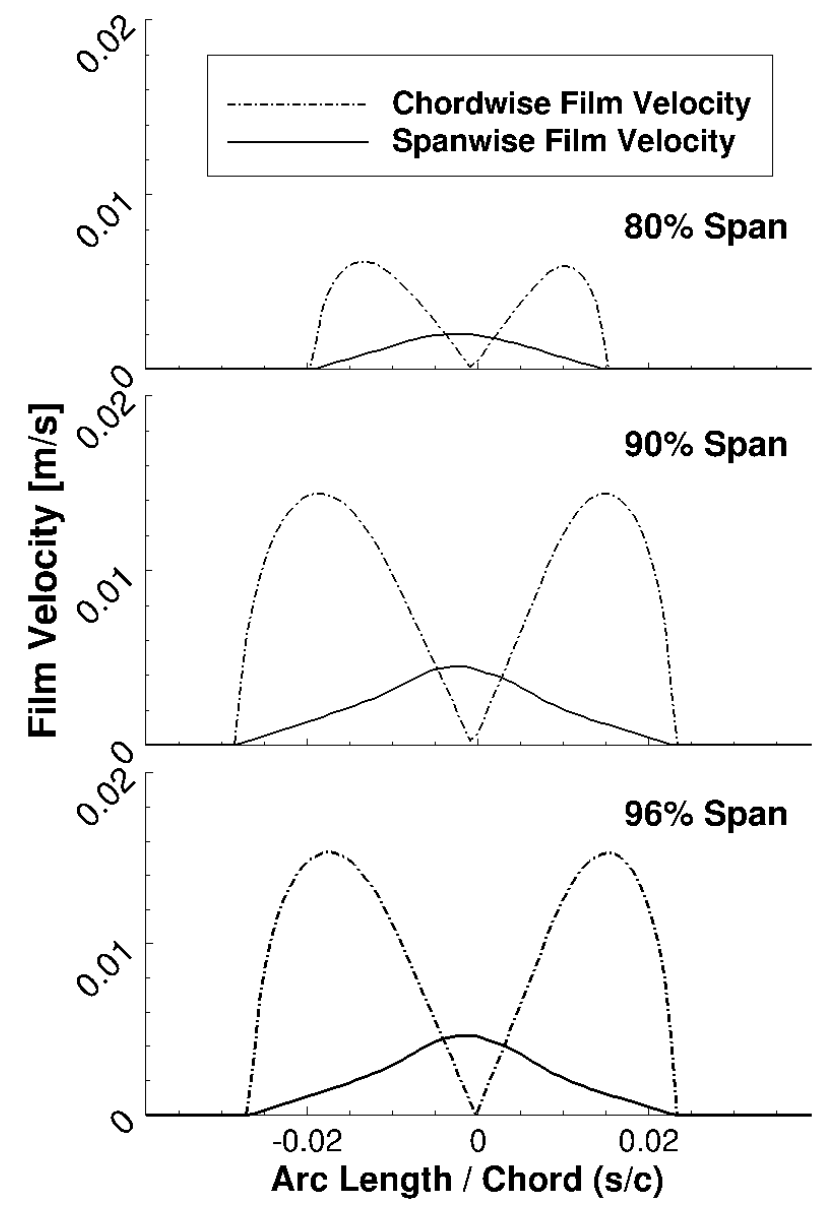

Fig. 7 Chordwise and spanwise water film velocity distribution, Hybrid 2D/3D approach

Resulting ice accretion shapes for select sections are shown in Fig. 8. Illustrated ice geometries were obtained using six ice accretion shots. The inboard section $(60 \%$ span) demonstrates negligible variations in ice geometry 
between the two approaches. At this inboard section, rime ice is occurring as no water film is present, therefore no major difference is expected between the two methods. The ice geometry has been marginally impacted by the quasi3D approach when mixed/glaze icing conditions are introduced at the mid-span and blade tip sections. At $80 \%$ and $90 \%$ span, a slight widening of the upper and lower horns is noted due to a migration of the water film near the stagnation point towards outboard sections as driven by the minimal centrifugal forces that characterize the rotor model. A slight reduction in sectional ice mass at the blade tip, illustrated in Fig. 9, is observed when using the Hybrid 2D/3D approach. This discrepancy is a result of the water film being pushed outwards towards the blade tip, where a sink condition has been placed to emulate water detachment. An increase in ice roughness values for the Hybrid 2D/3D approaches is also observed.
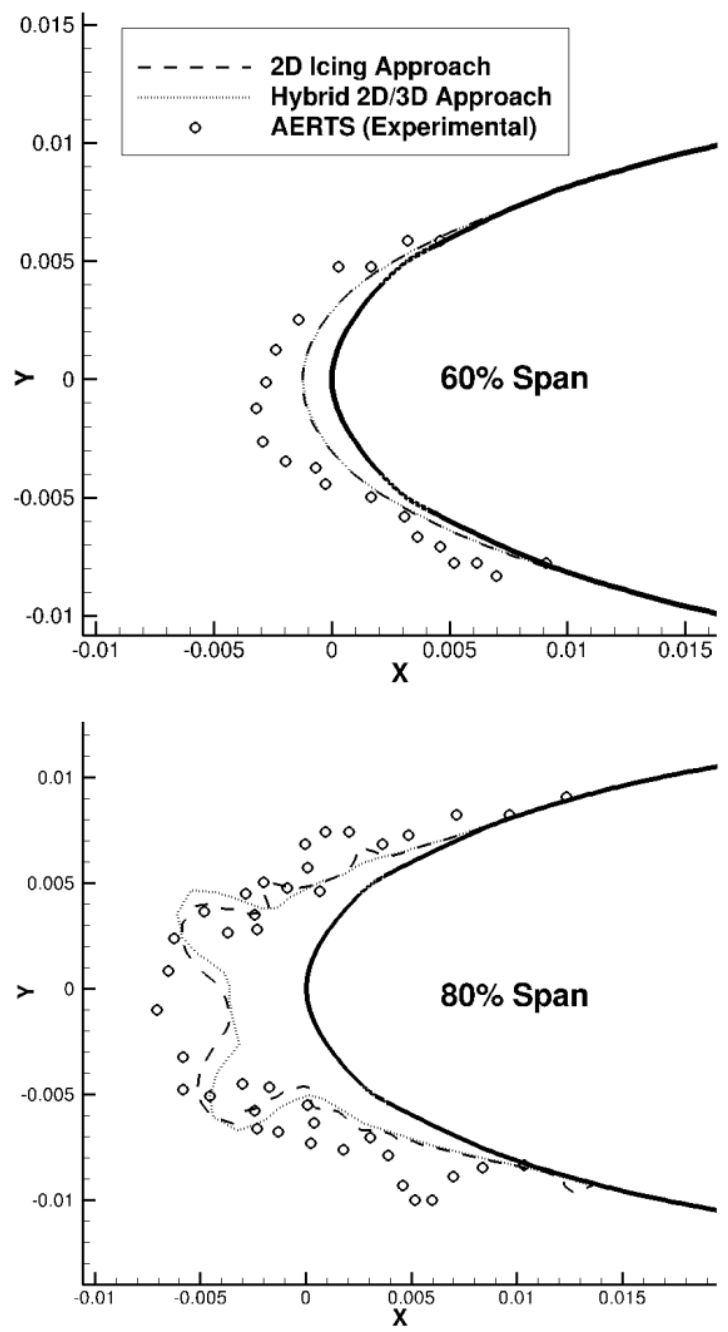


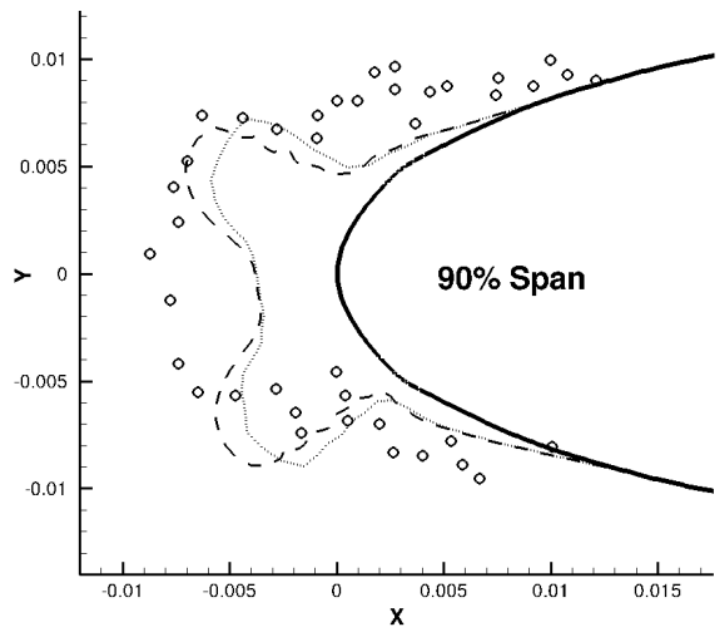

Fig. 8 Computed ice geometries at $60 \%, 80 \%$ and $90 \%$ (upper to lower) radius

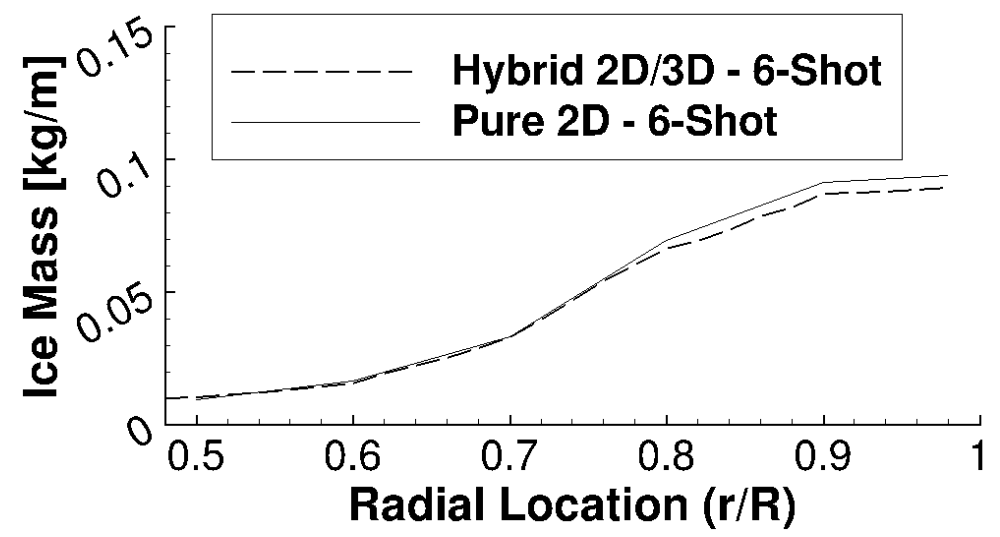

Fig. 9 Calculations for local mass of ice based on radial location, AERTS hover case

Processing times for the first ice accretion shot is summarized in Table 2. The values shown account for converged airflow / impingement solutions on all 24 blade sections. Calculations have been performed on Intel Xeon E5-2670 processors in a clustered environment. An increase in processing time due to the number of ice accretion shots is approximately linear.

Table 2 Processor time for the AERTS test case (Shot 1 of 6 ) 


\begin{tabular}{|l|c|c|c|c|}
\hline \multirow{2}{*}{\begin{tabular}{c}
\multirow{2}{*}{$\begin{array}{c}\text { Task / } \\
\text { Component }\end{array}$} \\
\cline { 2 - 5 }
\end{tabular}} & \multicolumn{2}{|c|}{ Processor Cost (Proc. $x$ Walltime) } \\
\cline { 2 - 5 } & HH:MM & $\%$ & \multicolumn{2}{c|}{ HHH:Mrid 2D/3D } \\
\hline $\begin{array}{l}\text { Airflow/Droplet } \\
\text { Steady Calc. }\end{array}$ & $194: 08$ & $99.8 \%$ & $194: 08$ & $99.8 \%$ \\
\hline $\begin{array}{l}\text { Solution / Mesh } \\
\text { Stitching }\end{array}$ & Nil. & Nil. & $0: 04$ & $0.03 \%$ \\
\hline $\begin{array}{l}\text { ICE3D Steady } \\
\text { Calculations }\end{array}$ & $0: 18$ & $0.16 \%$ & $0: 14$ & $0.12 \%$ \\
\hline \multicolumn{1}{|c|}{ TOTAL } & $194: 26$ & & $194: 26$ & \\
\hline
\end{tabular}

Solution and mesh stitching for the Hybrid 2D/3D case resulted in a 4 minute increase in processing time per ice accretion shot. However, the total processing time for ice accretion calculations is reduced slightly for the quasi-3D case. This is due to the solution read/write operations; in the pure 2D case, 24 separate ice accretion runs are performed, while the Hybrid 2D/3D approach performs one single ice accretion calculation per shot. Consequently, an identical processing time was found whether using $2 \mathrm{D}$ or quasi-3D approaches for this hover flight case.

\section{E. Rotor Degradation Results}

A 2D mesh was generated from the ice geometry at all radial sections using both approaches: 2D and quasi-3D. Degraded aerodynamic coefficients were obtained for the iced 2D geometries through an angle of attack sweep of each airfoil. The ice mass associated with each section has been calculated using an ice density of $917 \mathrm{~kg} / \mathrm{m}^{3}$. These were fed as inputs for the dynamics model, which was run through rotor speed and collective sweeps to evaluate the performance of the iced rotor.

A comparison of numerical and experimental results for the clean and ice-contaminated rotor at conditions representative of run 44 has been made. An under-prediction in ice-contaminated rotor torque rise is noted from the 2D icing approach. Conversely, the aerodynamic degradation associated with the hybrid 2D/3D process resulted in a torque rise of $9.2 \mathrm{Nm}$, or $127 \%$. This compares favorably to the $148 \%$ torque rise observed in the experiment. Despite the similarity in ice geometries in both approaches, the height and distribution of roughness calculated in the quasi3D approach yields performance degradation figures that better matched experimental results. A comparison of rotor torque for the iced and clean configurations, with correlations to experimental data when available, is provided in Fig. 10 and Fig. 11. 


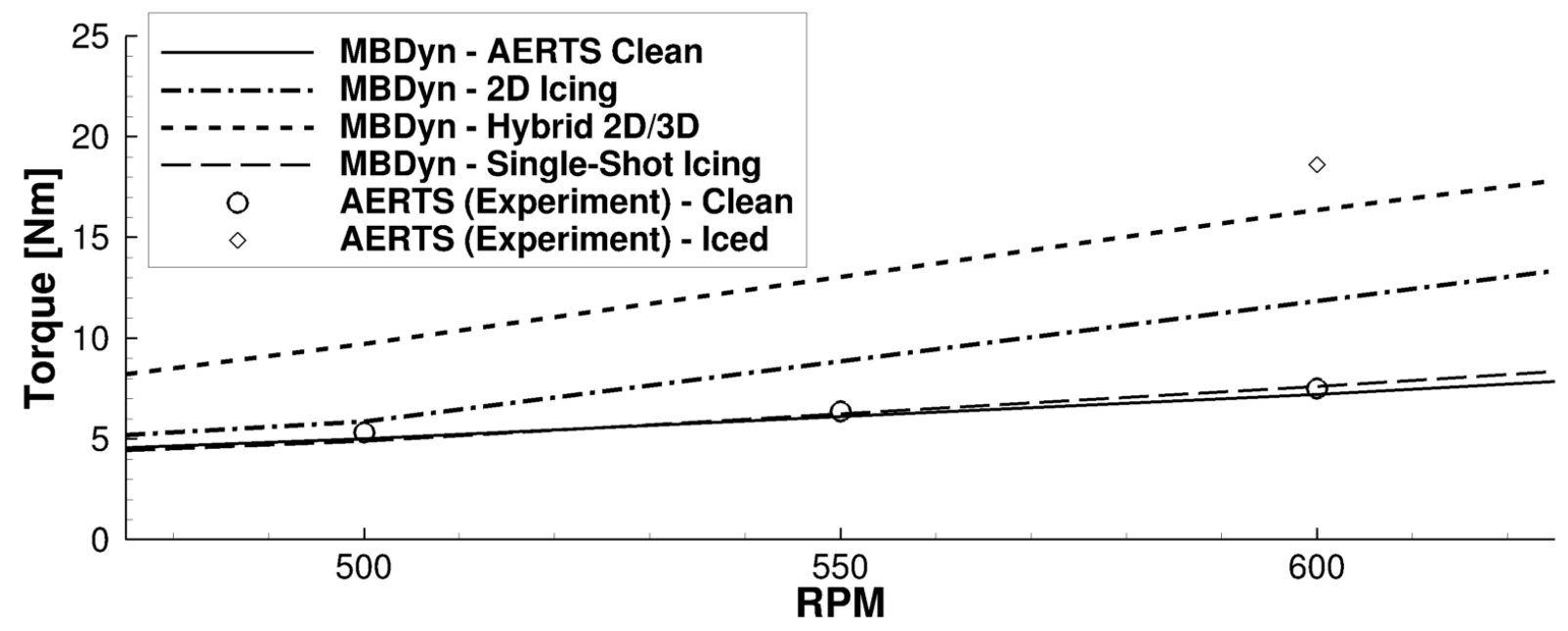

Fig. $10 \quad$ Iced rotor torque map, 2D and quasi-3D methods

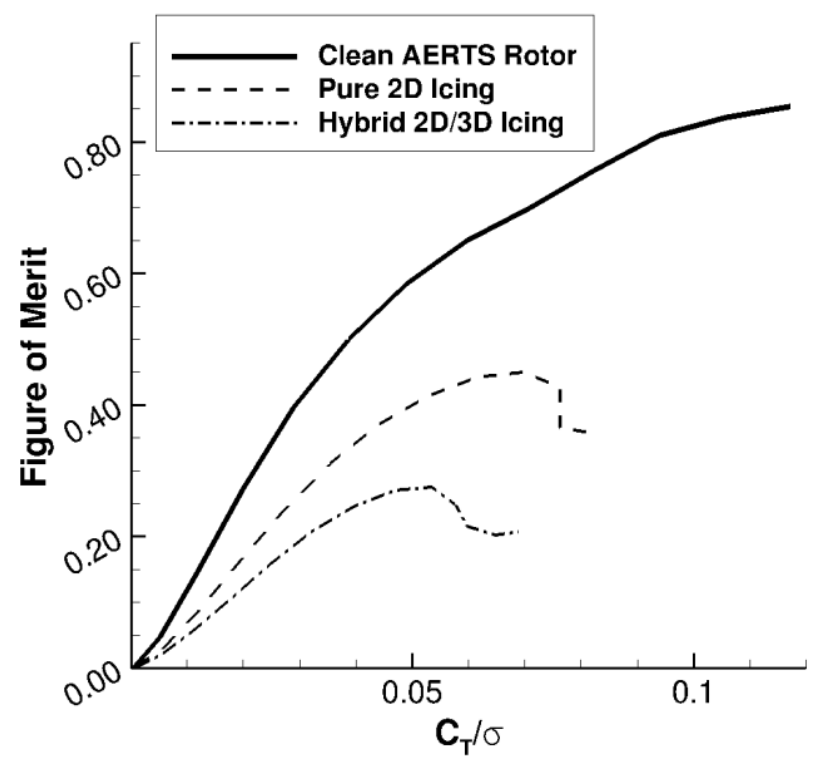

Fig. 11 FoM degradation due to icing on the AERTS rotor

\section{Forward Flight Case}

An experimental case for forward flight was selected from the testing of the Sikorsky Powered Force Model (SPFM) in the NASA Icing Research Tunnel (IRT) $[4,5]$. This work produced results for ice accretion performance effects on rotors through the use of an instrumented rotor and fuselage; a tail rotor was not present in the experiments. High rotor speeds in these tests achieved tip velocities and centrifugal forces comparable to a full-size rotor, making this a suitable evaluation case for the proposed simulation approach. The SPFM testing operated a 4-blade, fully 
articulated model-size rotor with a diameter of $1.86 \mathrm{~m}$. Hinges were located at $8.2 \%$ span, and a root cutout was present at $21.5 \%$. The rotor's rectangular blades featured an SC2110 airfoil profile, a chord of $0.1072 \mathrm{~m}$, and a linear twist of $11.5^{\circ}$

Flight condition ' 1 ' has been selected with a forward velocity of $40.7 \mathrm{~m} / \mathrm{s}$, at an advance ratio of 0.198 ; the environmental conditions encountered are representative of natural in-flight icing occurrences. Several experimental runs were performed at these conditions, and good repeatability in ice geometry and associated performance degradation were shown. Ice shedding has not been observed during these runs, and collective pitch has been held constant during the encounter. A summary of conditions and parameters are illustrated in Table 3.

Table 3 Experimental Conditions for Sikorsky PFM Test Condition '1'

\begin{tabular}{lcc}
\hline \multicolumn{1}{c}{ Parameter } & Value & Units \\
\hline Tip Speed & 205.7 & $\mathrm{~m} / \mathrm{s}$ \\
Advance Ratio & 0.198 & - \\
CL $_{\mathrm{\sigma}}$ & 0.064 & - \\
Static Temp. & -15.0 & ${ }^{\circ} \mathrm{C}$ \\
Shaft Angle & 2.6 & $\mathrm{deg}$ \\
LWC & 0.5 & $\mathrm{~g} / \mathrm{m}^{3}$ \\
MVD & 15 (monodisp.) & $\mu \mathrm{m}$ \\
Icing Time & 93 & $\mathrm{sec}$ \\
\hline
\end{tabular}

A model of the rotor was generated using fully-articulated rigid blades and an integrated swashplate. Collective and cyclic inputs were applied via pitch links. Aerodynamic performance data for the SC2110 airfoil is generated from 2D steady CFD solutions for angles of attack between -12 and +14 degrees. BEMT with the Drees linear inflow model has been used to calculate inflow distribution and rotor disc forces. An iterative approach has been implemented to balance rotor forces and reach trimmed conditions for collective pitch and cyclic inputs. The resulting angles of attack and Mach number distributions for the rotor disc are illustrated in Fig. 12. The torque and thrust values at the trimmed rotor condition were $1415 \mathrm{~N}$ and $59.3 \mathrm{Nm}$, respectively, compared to the $1416 \mathrm{~N}$ and $61.2 \mathrm{Nm}$ of the experimental case. 

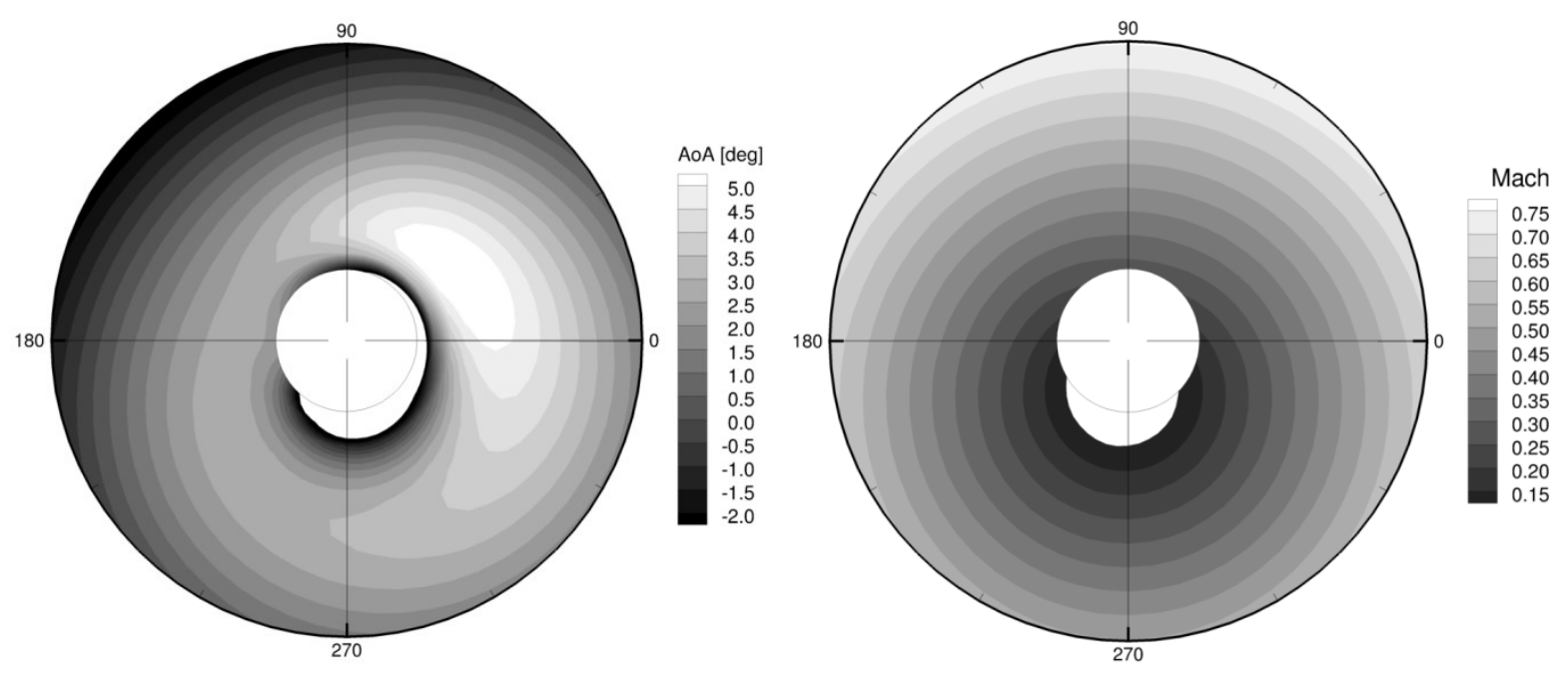

Fig. 12 Dynamic model inflow characteristics, AoA (left) and Mach (right), SPFM rotor

2D hybrid meshes have been generated at 22 blade sections, with 48,000 elements, and a maximum $\mathrm{y}^{+}$of 1 . A 3D surface mesh of the blade, with 17,300 elements, is generated from the 2D sections. Two views of a sample 2D hybrid mesh for the clean SC2110 airfoil are illustrated in Fig. 13.
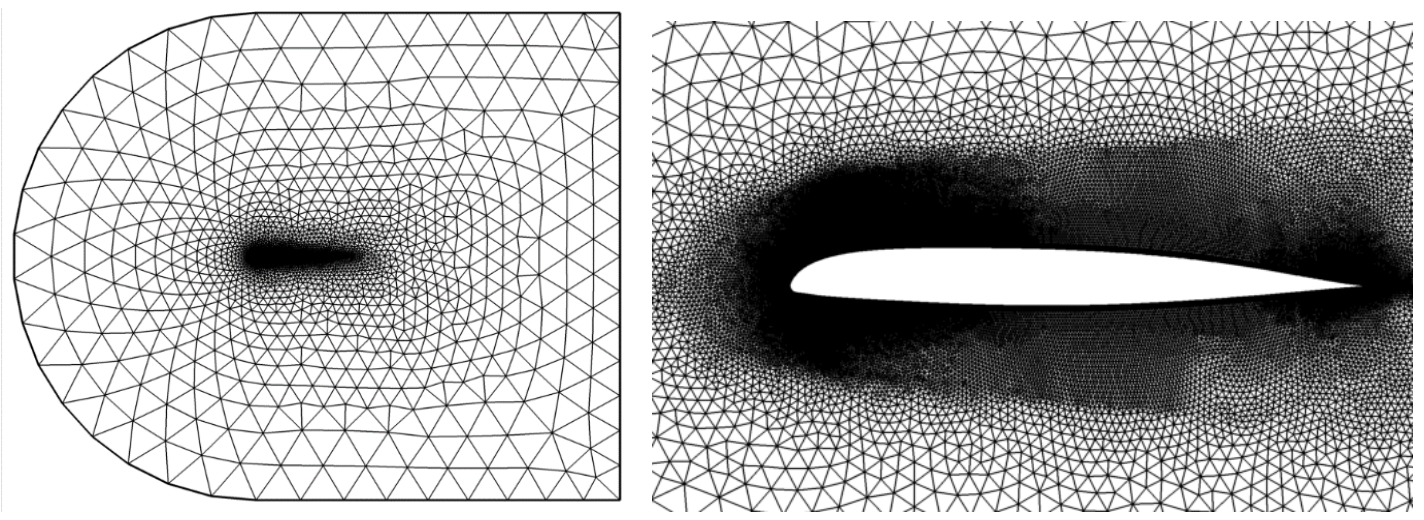

Fig. 13

Illustrations of the 2D hybrid SC2110 mesh [21]

Ice shapes for the 93 second encounter have been calculated using two strategies: a) an unsteady approach, using exclusively $2 \mathrm{D}$ calculations for ice accretion at various blade sections, and b) the unsteady hybrid 2D/3D approach, using a quasi-3D approach for ice accretion sourced through unsteady simulations. Each approach is evaluated using one, three and six shots.

\section{Aeroicing and Performance Results}


Spanwise and chordwise water film velocities are shown for the first shot at three sections in Fig. 14. No water film is predicted inboard of $0.79 \mathrm{~m}(85 \%)$ span, aggravated by the low temperatures and small droplet diameters in the experiment. Mixed or glaze icing is encountered in the blade tip region due to aero-thermal heating; correspondingly, the quasi-3D approach had the greatest effect near the blade tip. Film velocity is shown to increase towards the tip region as glaze icing is exhibited. The greatest value for spanwise film velocity is noted near the stagnation point, where aerodynamic shear stress is low.

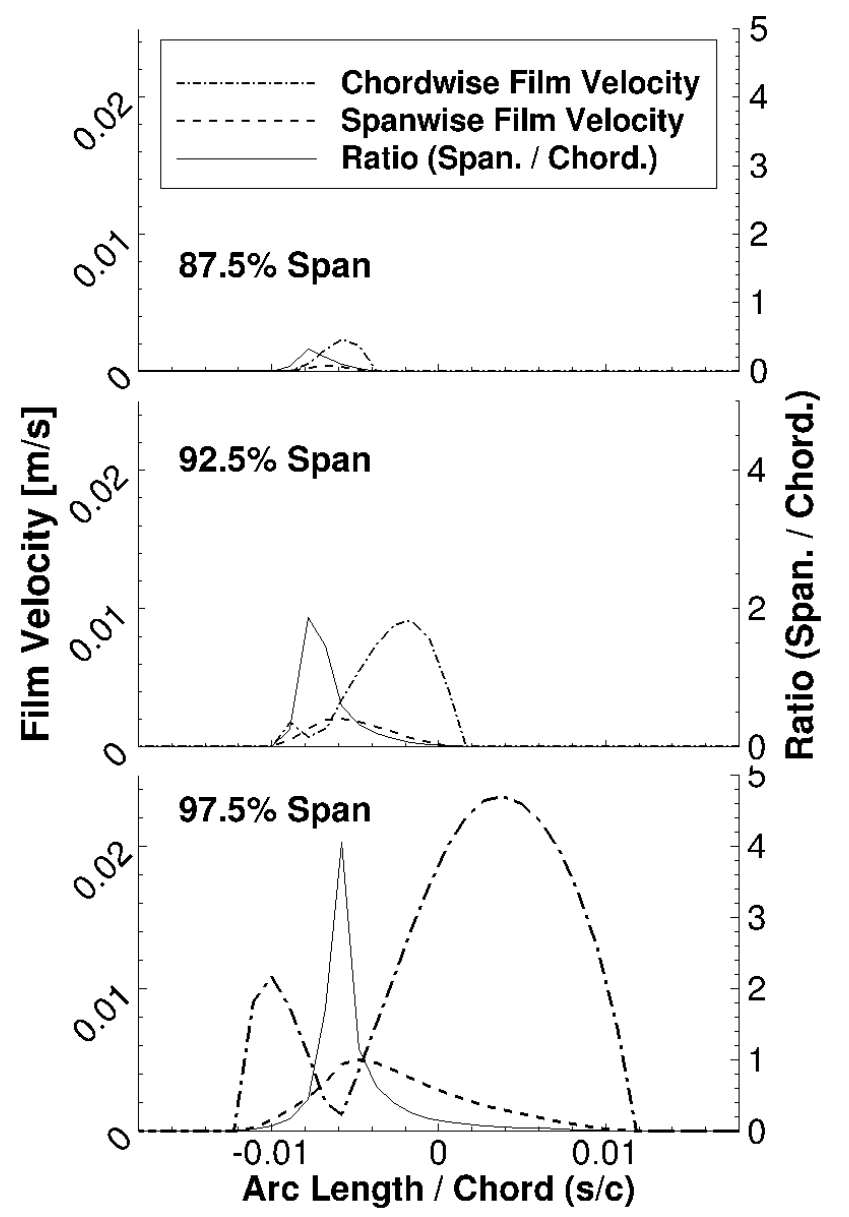

Fig. 14 Chordwise and spanwise water film velocity distribution, SPFM rotor

Water film thickness for select radial sections is illustrated in Fig. 15. Significant discrepancies are noted between the $2 \mathrm{D}$ and hybrid $2 \mathrm{D} / 3 \mathrm{D}$ water film thicknesses in this test case, due to the high incident and rotational velocity encountered near the tip. Here, the water film is shown to pool near the blade tip, resulting in an increase in water film thickness as shown by the hybrid 2D/3D simulation. 


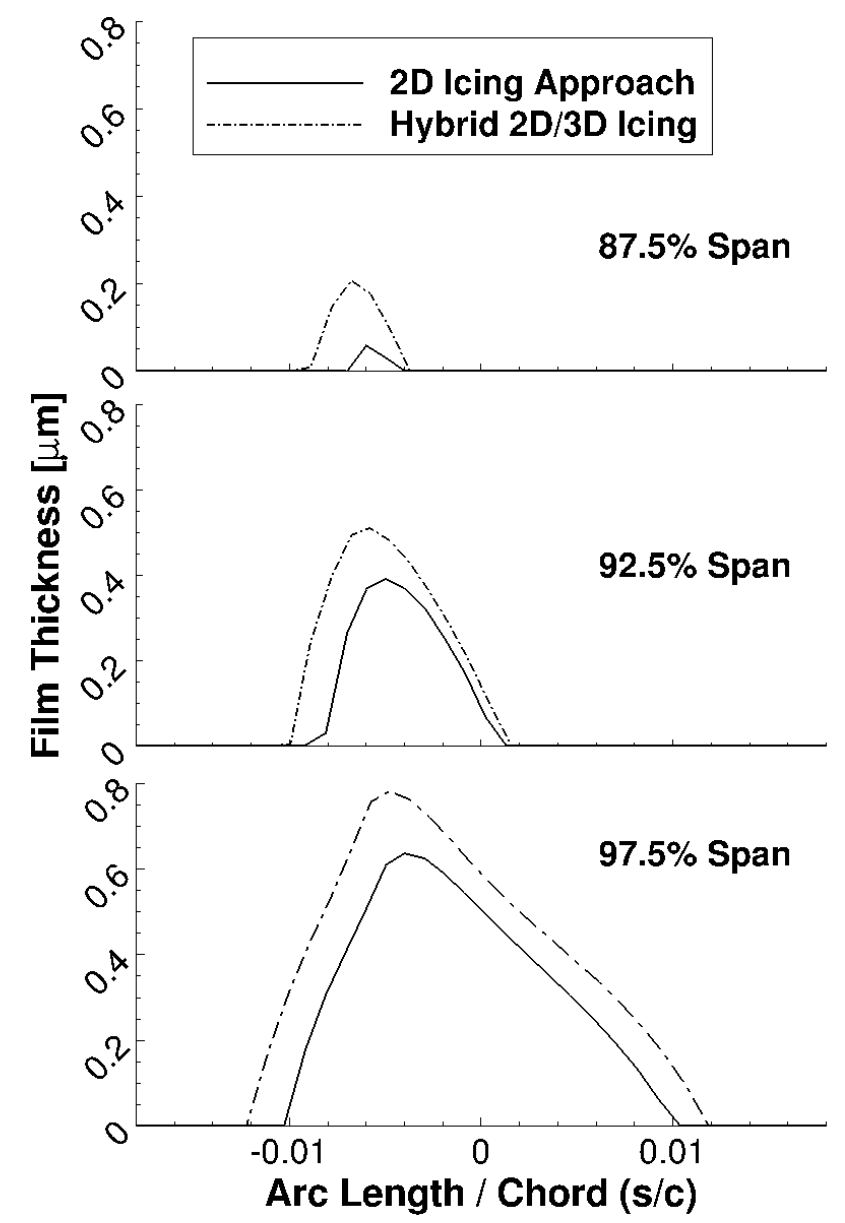

Fig. 15 Water film thickness distribution, SPFM rotor

Ice shapes for each approach, and number of shots, were compared to the experimental data available; a visual comparison at select radial sections is provided. At 50\% and 70\% span, both approaches demonstrated comparable results and fair ice shape prediction when compared to experiments. This can be attributed to the low incident velocity at these sections and to the local rime icing conditions. Near the blade tip ( $90 \%$ span), the use of the quasi-3D approach demonstrates improvements over 2D approaches. A better prediction of the angle of the upper and lower horns, as well as the valley between horns, is observed for the 6-shot Hybrid 2D/3D results in Fig. 17. However, both approaches do not capture the full extent of icing, which may be a limitation of the 2D approach presented. Regardless, the authors believe an improvement in ice geometry results are observed due to the use of additional shots and the Hybrid 2D/3D approach (when compared to previous 2D approaches), especially near the blade tip. 

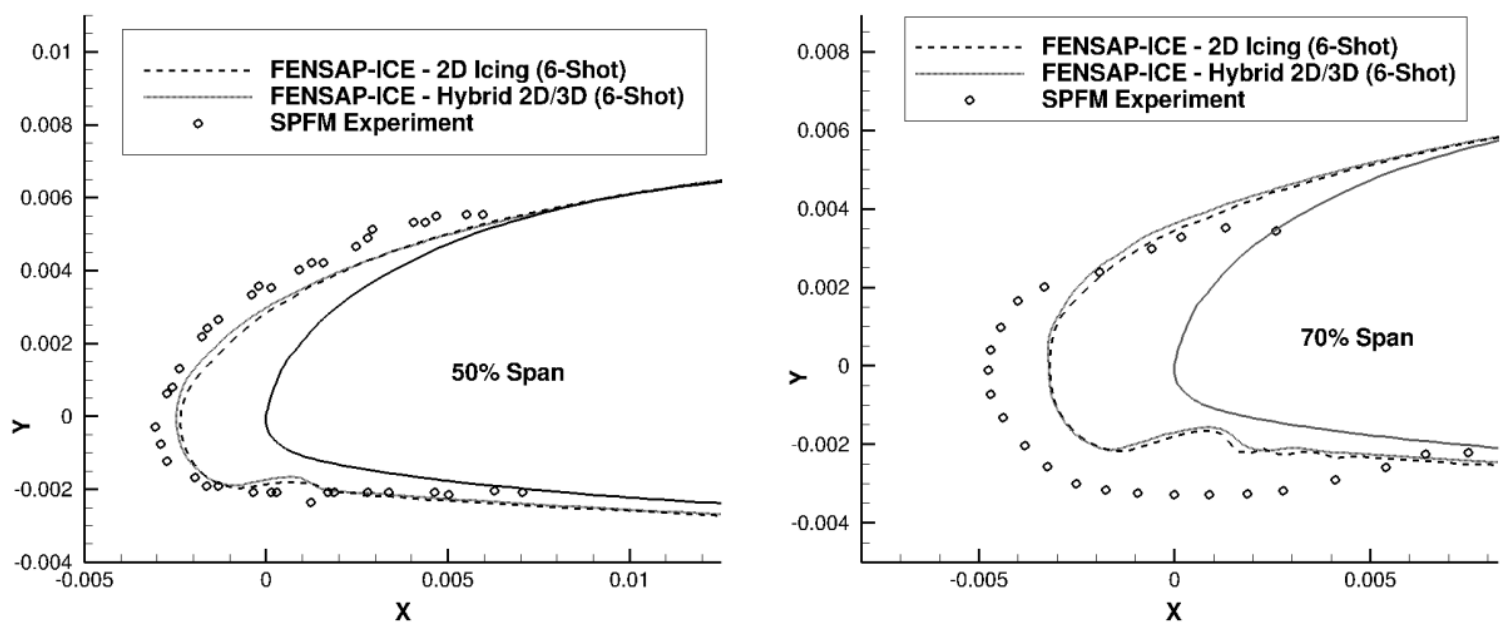

Fig. 16 Computed ice geometries at 50\% (left) and 70\% (right) span, 6 icing shots
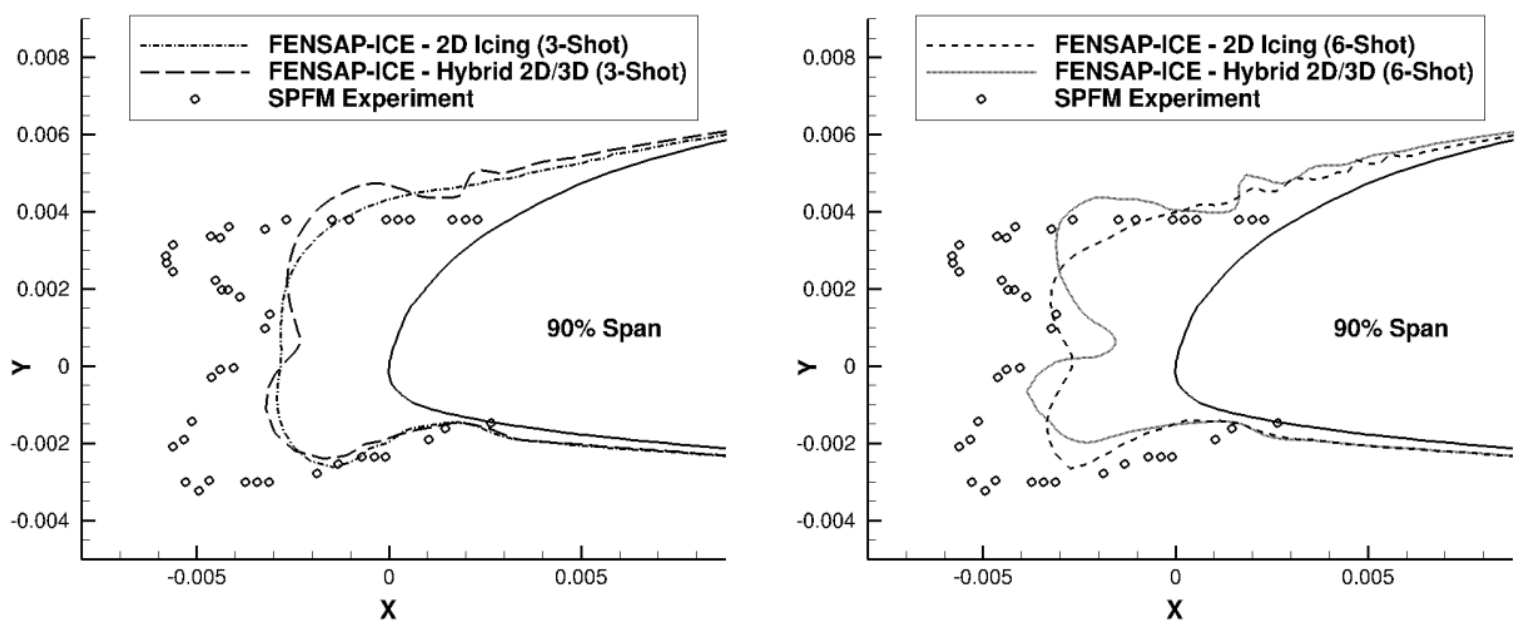

Fig. 17 Computed ice geometry at 90\% span via 2D and quasi-3D methods, 3 (left) and 6 (right) icing shots

Results for the first ice accretion shot in the 6-shot evaluation are summarized in Table 4. Reference steady and unsteady airflow and droplet impingement calculations are identical for both the $2 \mathrm{D}$ and Hybrid $2 \mathrm{D} / 3 \mathrm{D}$ results. The Hybrid approach requires an additional 2 processor-hours to perform the mesh and solution stitching, while a slight increase in processing time for unsteady icing calculations is noted. The additional processing cost attributed to using the Hybrid 2D/3D approach is approximately 3 hours, or $0.8 \%$, in this case. 
Table 4 Processor Times for the SPFM Case (Shot 1 of 6)

\begin{tabular}{|l|r|r|r|c|}
\hline \multirow{2}{*}{\begin{tabular}{c}
\multirow{2}{*}{$\begin{array}{c}\text { Task } / \\
\text { Component }\end{array}$} \\
\cline { 2 - 5 }
\end{tabular}} & \multicolumn{2}{|c|}{ Processor Cost (Proc. $x$ Walltime) } \\
\cline { 2 - 5 } & HHH:MM & $\%$ & \multicolumn{2}{c|}{ HHH:Mrid 2D/3D } \\
\hline $\begin{array}{l}\text { Airflow/Droplet } \\
\text { Steady Calc. }\end{array}$ & $111: 48$ & $28.2 \%$ & $111: 48$ & $28.0 \%$ \\
\hline $\begin{array}{l}\text { Airflow/Droplet } \\
\text { Unsteady Calc. }\end{array}$ & $279: 30$ & $70.5 \%$ & $279: 30$ & $70.0 \%$ \\
\hline $\begin{array}{l}\text { Solution / Mesh } \\
\text { Stitching }\end{array}$ & Nil. & Nil. & $2: 00$ & $0.50 \%$ \\
\hline $\begin{array}{l}\text { ICE3D Steady } \\
\text { Calculations }\end{array}$ & $4: 53$ & $1.24 \%$ & $6: 04$ & $1.52 \%$ \\
\hline \multicolumn{1}{|c|}{ TOTAL } & $396: 11$ & & $399: 22$ & \\
\hline
\end{tabular}

\section{F. Rotor Degradation Results}

A steady-state aerodynamic sweep of angles from -12 to +14 degrees is performed. Ice mass properties are extracted and added as point masses to the dynamic model. To simulate the constant collective pitch maintained in the encounter, the pre-icing control inputs were held constant and the losses in thrust / gains in torque were noted. The performance degradation for each of the icing sequences tested are summarized in Table 5 and Fig. 18:

Table 5 Icing Performance Degradation Summary for Forward Flight Model

\begin{tabular}{|c|r|r|r|r|r|r|}
\cline { 2 - 8 } \multicolumn{1}{c|}{} & \multicolumn{2}{c|}{ Performance Values } & \multicolumn{4}{c|}{ Performance Degradation } \\
\hline \multirow{2}{*}{ Configuration } & \multicolumn{1}{c|}{ Thrust } & \multicolumn{1}{c|}{ Torque } & \multicolumn{2}{c|}{ Thrust } & \multicolumn{2}{c|}{ Torque } \\
& $\mathbf{N}$ & \multicolumn{1}{c}{$\mathbf{N m}$} & \multicolumn{1}{c|}{$\mathbf{N}$} & \multicolumn{1}{c|}{$\%$} & \multicolumn{1}{c|}{ Nm } & \multicolumn{1}{c|}{$\%$} \\
\hline SPFM - Clean (Exp.) & 1416.3 & 61.19 & N/A & N/A & N/A & N/A \\
\hline SPFM - Config. 1 (Exp.) & 1238.3 & 105.16 & -178.00 & $-12.6 \%$ & 43.97 & $71.9 \%$ \\
\hline \hline MBDyn Model - Clean & 1414.8 & 59.26 & N/A & N/A & N/A & N/A \\
\hline 2D Unsteady, 1-Shot & 1410.8 & 63.39 & -4.0 & $-0.3 \%$ & 4.1 & $7.0 \%$ \\
\hline 2D Unsteady, 3-Shot & 1385.0 & 87.68 & -29.8 & $-2.1 \%$ & 28.4 & $48.0 \%$ \\
\hline 2D Unsteady, 6-Shot & 1378.2 & 94.29 & -36.6 & $-2.6 \%$ & 35.0 & $59.1 \%$ \\
\hline Hybrid 2D/3D, 1-Shot & 1406.0 & 62.39 & -8.8 & $-0.6 \%$ & 3.1 & $5.3 \%$ \\
\hline Hybrid 2D/3D, 3-Shot & 1385.0 & 89.03 & -29.8 & $-2.1 \%$ & 29.8 & $50.2 \%$ \\
\hline Hybrid 2D/3D, 6-Shot & 1369.5 & 94.07 & -45.3 & $-3.2 \%$ & 34.8 & $58.7 \%$ \\
\hline
\end{tabular}




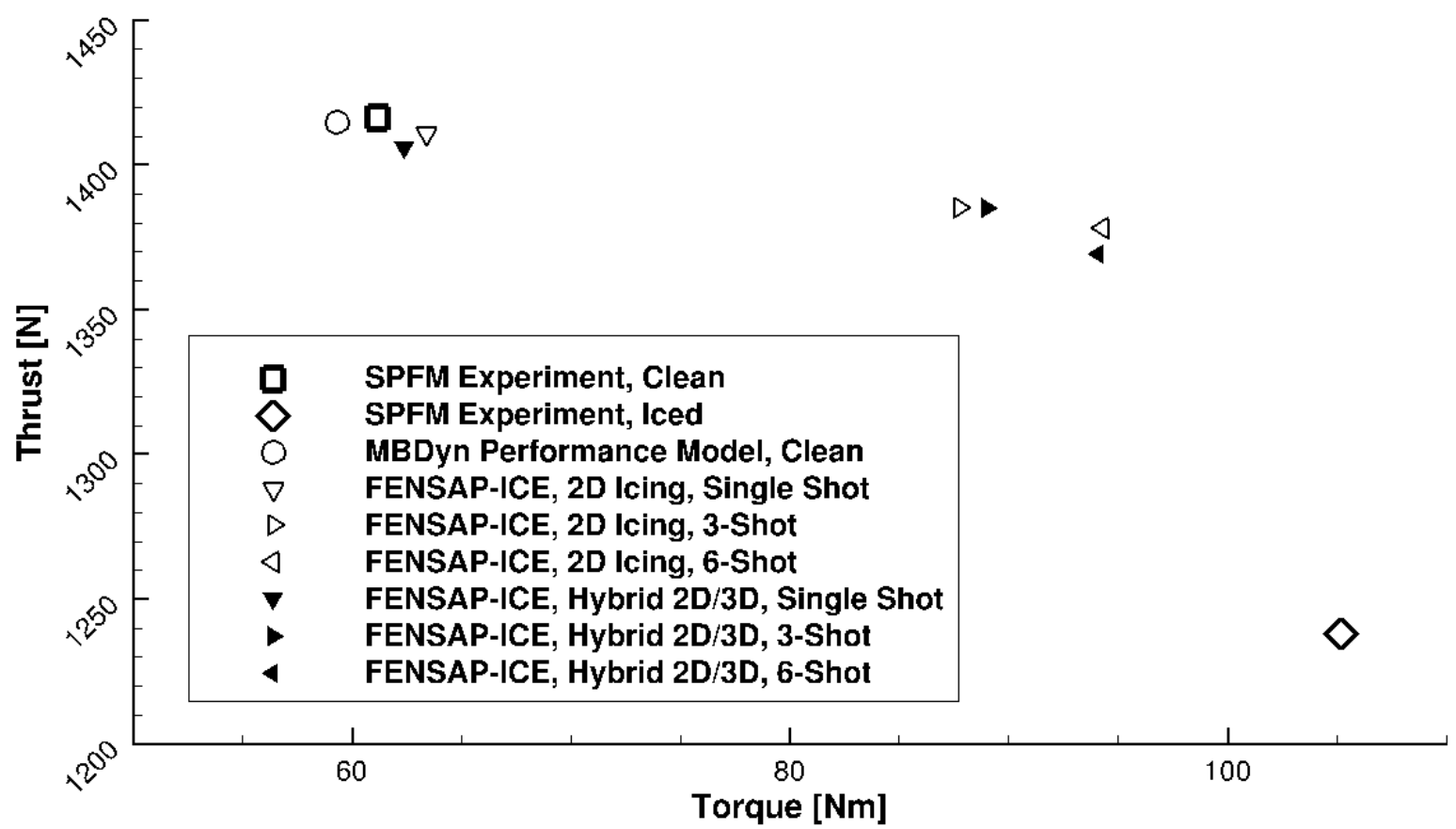

Fig. 18 Rotor performance summary via experimental and numerical approaches, SPFM rotor

Using either approach, increasing the number of ice accretion shots provides a more accurate prediction of torque rise, and a slight improvement in thrust loss predictions. In this particular case, the use of the Hybrid 2D/3D approach demonstrates similar rises in torque when compared to the geometries obtained through entirely $2 \mathrm{D}$ methods. For the 6-shot sequence, both strategies are a fair match to torque rise, predicting a $59 \%$ rise compared to a $72 \%$ rise in the experiment. All sequences tested under-predicted thrust loss values. It is noted the Hybrid 2D/3D approach has slight improvements in thrust loss predictions for the 6-shot case.

The similarities in predicted ice geometries predicted through the 2D and Hybrid 2D/3D approaches are illustrated in Fig 16 and Fig. 17. The primary advancement of the Hybrid 2D/3D approach is the consideration of spanwise water film motion when in glaze icing conditions; therefore, only after the transition from rime to mixed/glaze icing at $85 \%$ is a significant discrepancy between ice geometries noted. This is in contrast to the hover flight case, where the higher temperature and liquid water content yielded mixed or glaze icing along a greater length of the blade.

Using either the 2D or Hybrid 2D/3D approach, the computed ice geometries were smaller than experimental results. When analyzing the aerodynamic losses at individual blade sections, the degradation of the computational geometries were not as significant as the experimental shapes. Correspondingly, an insufficient loss in thrust is 
predicted. Nevertheless, the aggressive double-horn geometries at $90 \%$ span are better predicted by using the Hybrid 2D/3D approach with 6 icing shots. Examining the local aerodynamic characteristics of the blade at $90 \%$ span illustrate the differences in overall rotor performance characteristics:

The hemispherical-bead model calculates roughness height based on aerodynamic shear stress, gravity forces and the pressure gradient along the blade profile [14]. The high tip velocity on the SPFM rotor contributes to a high aerodynamic shear stress component on the blade, reducing the roughness of ice when compared to the AERTS rotor. Thus the discrepancy between the roughness profiles encountered using $2 \mathrm{D}$ and Hybrid 2D/3D approaches is further minimized in this forward flight case, yielding similar performance results when using either of the two approaches.

\section{Conclusions}

A cost-effective numerical approach addressing 3D icing over helicopter blades has been proposed for the evaluation of performance degradation of isolated rotors due to in-flight ice accretion in hover and forward flight conditions. The methodology seeks a compromise between accuracy in predicting performance degradation and computational complexity that makes it suitable for early and preliminary design phases of the rotor and the associated IPS. The process implements a loose coupling between a multibody approach to rotor dynamics and an advanced CFD-icing approach to thoroughly analyze the ice accretion process and associated performance degradation.

The proposed methodology allows considering any rotor geometry for ice accretion in a computationally efficient manner. 2D airflow and droplet impingement calculations are interpolated into 3D solution files as part of the aeroicing process. The three-dimensional ice accretion considers 3D heat transfer and water runback effects on a rotating blade. The framework has been applied to wind tunnel test cases for rotors in hover and forward flight. The hybrid 2D/3D approach captures the presence of a spanwise water film motion otherwise neglected in a purely 2D approach. Considering this water film motion shows general improvements in ice geometry and performance prediction. Fair agreement with experimental values for torque rise are noted in hover and forward flight test cases, with improvements obtained, as expected, by increasing in number of shots in the multishot approach. An evaluation of thrust and torque values for the iced rotors reveals the hybrid 2D/3D approach to consistently yield more conservative, 'worst-case' rotor performance degradation. However, an under-prediction in performance degradation is still noted when comparing the hybrid approach with experimental results, especially when predicting thrust loss in the forward flight case. This may be due to some limitations in the 2D aerodynamic calculations for what concerns turbulence modeling 
and impact on the multi-phase flow of air and droplets. One other relevant source of discrepancy between the simulations and available experimental data could also be the due to the necessity of addressing the interaction between an undisturbed flow of droplets hitting the blade section and the perturbed droplet flow coming from the preceding blade. Alteration in terms of droplet distributions and velocity due to such an interaction might affect the water deposition and subsequent icing along the blade span.

Nevertheless, the hybrid 2D/3D approach yields what could be considered as conservative values characterized by a good matching in terms of the topological characteristics of the resulting ice shape, i.e. number of horns, type of icing and a fair agreement in terms of detailed geometrical aspects of the accreted ice with the available experimental results. It is noted however that such discrepancies on the details of the ice shapes impact the predictions in terms of rotor performance degradation. Realizing a detailed matching between experimentally measured ice shapes and numerically computed solutions is quite challenging due to the limitations of both approaches in such a complex multiphase flows. Further advances in modeling and experimental techniques are clearly recognized by the scientific community and many efforts are pout in this direction. Nevertheless, in the case of the present work, the focus was more on a methodology for early design able to take into consideration not only aeroicing aspects but also aspects related to the changes that ice induces on the dynamical characteristics of the rotor blades, therefore it has been possible on one side to release some strict constraints on realizing a perfect matching between numerical and experimental results but on the other side to seek for improvement over currently used reduced dimensionality/fidelity approaches.

The application of the hybrid approach is shown to negligibly affect the overall computational cost of a purely $2 \mathrm{D}$ aeroicing simulation. This consideration might appear trivial given that the simulation of the airflow and droplet dynamics is usually the most demanding part of the calculation. Nevertheless, it should be observed that in this case, simulating a pure $3 \mathrm{D}$ icing process requires a mesh which is between 20 and 30 times bigger than what would be used in a pure 2D case and the computational cost of the icing part do not scale linearly with the number of nodes in the surface mesh.

The experimental cases presented in this work illustrate scaled rotor geometries and short-scale tests of 3 minutes or less. In these cases, the shedding of ice from the rotor blade was not observed. When considering real-world operations, the geometry of full-size rotors and the potential for significantly longer exposure times can frequently lead to the shedding of ice from the blades. A predictive shedding model would improve the accuracy of ice geometry 
prediction over long-scale icing encounters, and forms a major focus of future work by the present group. A tighter coupling of multi-body dynamics and CFD-icing modules is also desired to better account for aeroelasticity impacts on the ice accretion process.

With future advancements, the dynamic icing model for rotor performance has an enormous potential for safety in the helicopter industry. As a large assortment of conditions and geometries may be evaluated in a short time period, the relative cost of IPS development can be reduced compared to wind tunnel testing. A model of this type, when coupled with an optimization code, can allow for rotorcraft IPS design to reach an ideal compromise between competing design factors.

\section{Acknowledgments}

The authors of this paper would like to thank Habibollah Fouladi and Sahar Choukir of the McGill CFD Laboratory for their contributions to the unsteady aeroicing process. James Corrigan and Roger Aubert from Bell Helicopter Textron for their highly valued technical comments. This work was conducted as part of the NSERC / Lockheed Martin / Bell Helicopter Research Chair for Multi-disciplinary Analysis and Design of Aerospace Systems. Computations were made on the supercomputer Guillimin from McGill University, managed by Calcul Québec and Compute Canada.

\section{References}

1. Peterson, A. A., Dadone, L., and Bevan, D. "Rotorcraft aviation icing research requirements: Research review and recommendations." NASA CR-165344, May 1981.

2. Flemming, R. J., and Lednicer, D. A. "High Speed Ice Accretion on Rotorcraft Airfoils." NASA Contractor Report $3910,1985$.

3. Brouwers, E. W. "The Experimental Investigation of a Rotor Icing Model with Shedding." Pennsylvania State University, Thesis, May 2010.

4. Britton, R., Bond, T., and Flemming, R. J. "An Overview of a Model Rotor Icing Test in the NASA Lewis Icing Research Tunnel." NASA TM 106471, 1994.

5. Britton, R. K. "Ice Accretion Characteristics of a Model Rotor in the NASA Lewis Icing Research Tunnel," AHS/SAE International Icing Symposium '95. Montreal, October 18-21, 1995.

6. Kreeger, R. E., Sankar, L., Narducci, R., and Kunz, R. "Progress in Rotorcraft Icing Computational Tool Development." SAE Technical Paper 2015-01-2088, 2015.

7. Habashi, W. G. "Recent Advances in CFD for In-Flight Icing Simulation," Japan Society of Fluid Mechanics Vol. 28, No. 2, 2009, pp. 99-118. 
8. Rajmohan, N., Bain, J., Nucci, M., Sankar, L., Flemming, R., Egolf, T. A., and Kreeger, R. "Icing Studies for the UH-60A Rotor in Forward Flight," AHS Aeromechanics Specialists' Conference. 2010.

9. Narducci, R., and Kreeger, R. E. "Analysis of a Hovering Rotor in Icing Conditions." NASA TM-2012-217126, Jan 2012.

10. Narducci, R., Orr, S., and Kreeger, R. E. "Application of a High-Fidelity Icing Analysis Method to a Model-Scale Rotor in Forward Flight." NASA TM-2012-217122, Jan 2012.

11. Kelly, D., Fouladi, H., Fossati, M., Habashi, W., Alicino, R., Quaranta, G., and Masarati, P. "Assessment of Ice Accretion Effects on Rotor Dynamics via Multi-Body and CFD Approaches," AHS 70th Annual Forum. Montreal, Canada, 2014.

12. Masarati, P., Morandini, M., and Mantegazza, P. "An Efficient Formulation for General-Purpose Multibody/Multiphysics Analysis," ASME J. Comput. Nonlinear Dyn Vol. 9, No. 4, October 2014.

13. Leishman, J. G. Principles of Helicopter Aerodynamics. New York, NY: Cambridge University Press, 2000.

14. Croce, G., De Candido, E., Habashi, W. G., Munzar, J., Aube, M. S., Baruzzi, G. S., and Aliaga, C. "FENSAPICE: Analytical Model for Spatial and Temporal Evolution of In-Flight Icing Roughness," Journal of Aircraft Vol. 47, No. 4, 2010.

15. Beaugendre, H., Morency, F., and Habashi, W. G. "FENSAP-ICE's Three-Dimensional In-Flight," Journal of Aircraft Vol. 40, No. 2, 2003.

16. Bourgault, Y., Habashi, W. G., Dompierre, J., and Baruzzi, G. S. "A finite element method study of Eulerian droplets impingement models," International Journal for Numerical Methods in Fluids Vol. 29, No. 4, 1999, pp. 429-449.

17. Tanner, W. H., and Yaggy, P. F. "Experimental Boundary Layer Study on Hovering Rotors," Journal of the American Helicopter Society Vol. 11, No. 3, July 1966, pp. 22-37.

18. McCroskey, W. J. "Measurements of Boundary Layer Transition, Separation and Streamline Direction on Rotating Blades." NASA TN D-6321, 1971.

19. Fouladi, H., and Habashi, W. G. "FENSAP-ICE: Simulation of ice accretion on an oscillating airfoil," SAE AeroTech Congress \& Exhibition. Montreal, Canada, 2013.

20. Brouwers, E. W., Palacios, J. L., Smith, E. C., and Peterson, A. A. "The Experimental Investigation of a Rotor Hover Icing Model with Shedding," American Helicopter Society 66th Annual Forum. Phoenix, AZ, May 2010.

21 Dussin, D., Fossati, M., Guardone, A., and Vigevano, L. "Hybrid grid generation for two-dimensional highReynolds flows," Computers \& Fluids, Vol. 38, No. 10, 2009, pp. 1863-1875. 